\section{Another One for the Book}

Punters betting on the molecular biology handicap, where scientific reputations are at stake, have taken a trouncing in the past few months. First the central dogma, or, to be more precise, the popular misconception of the central dogma, was shown to be an oversimplification. Now another firm favourite has been shaken. On page 227 of this issue of Nature, Chamberlin and his colleagues report, against all the betting, that when the bacteriophage 'T7 infects $E$. coli, the phage genome specifies a complete RNA polymerase molecule, rather than a sigma factor with the capacity to subvert the pre-existing RNA polymerase of the bacterium to the transcription of the phage genome.

In $E$. coli, RNA polymerase comprises the so-called core enzyme, which catalyses the formation of phosphodiester bonds between successive nucleotides of an RNA molecule, and a sigma factor, which programmes the core enzyme to transcribe only certain genes. The sigma factor transiently associates with the core and the complex is able to recognize the initiation sequences of certain genes. Once the initiation of transcription has been achieved, the sigma factor dissociates and is free to programme another core molecule, while the core, once it has completed the round of transcription, is free to associate with a different sigma factor. This mechanism seems to be an economical way of providing RNA polymerase with specificity, and, assuming a battery of different sigma factors and a set of core molecules, it is easy enough to devise schemes to account for the regulation of gene expression.

As soon as the first sigma factor of $E$. coli had been discovered, there was speculation that bacteriophages might bring about the transcription of the phage genome, at the expense of the transcription of the bacterial genome, by specifying one or more species of sigma factors which are able to initiate the transcription of phage DNA but not bacterial DNA. This scheme has two prerequisites: first the phage sigma factor must be able to displace host sigma factors and, second, the phage gene which specifies the first species of phage sigma factor must itself be recognized by the core enzymo complexed with a bacterial sigma factor.

Chiefly as a result of the work of Travers and his collaborators and Bautz and his colleagues, we know that the transcription of phage T4 genomes in $E$. coli follows this pattern. One of the first products of transcription and translation of the phage genome is a phage sigma factor which then allows the transcription of other phage genes. Furthermore, in the later stages of the infection, a second phage protein, which may well be another sigma factor, is made and this permits the expression of the late genes of the phage. With the precedent of $\mathrm{T} 4$ in mind it was not surprising that Summers and Siegel (Nature, 223, 1111; 1969) assumed they had discovered yet another sigma factor when they found that the protein specified by gene 1 of phage $T 7$ is made early in infection and seems to be required for the transcription of the T7 genome. What made this interpretation particularly seducing was the finding that in vitro the gene 1 protein transcribed the DNA of phage T7 to a much greater extent in the presence of $E$. coli RNA polymerase than in its absence.

But there were two flies in this balm: Summers and Siegel found that, in vitro at least, the product of gene 1 of $\mathrm{T} 7$ phage was unable to displace host sigma factor from core, and, more importantly, T7 infections were found to be insensitive to rifamycin, a drug which inhibits $E$. coli RNA polymerase. At that time the first of these findings seemed unimportant because, although the putative T7 sigma factor failed to displace host sigma factor, the product of gene 1 seemed to bind to core enzyme of $E$. coli. The second observation raised a more awkward question: how do T7 infections proceed normally in the presence of rifamycin if the core enzyme of $E$. coli, which is sensitive to this drug, is involved in the transcription of the phage genome? Summers glossed over this objection by arguing that because T7 messenger RNAs are unusually stable, and assuming that the whole of the T7 genome is transcribed very soon after infection, the presence of rifamycin rapidly becomes immaterial.

Clearly Chamberlin, with perspicacity, was unconvinced, believing that the failure of rifamycin to block T7 infections was highly significant. He and his colleagues isolated the product of gene 1 of $T 7$ and found that it was a complete RNA polymerase capable of transcribing the T7 genome, and physically and biochemically quite distinct from the host enzyme. Furthermore, the T7 enzyme is resistant to rifamycin. With hindsight it is easy to see why Summers and Siegel, along with most people including some of the mandarins, were led astray, especially at a time when sigma factors were being invoked to explain every and any example of controlled gene expression. It was their misfortune to be dealing with a protein which has one particularly unusual property. T7 RNA polymerase loses its activity in dilute solutions unless it is stabilized by some other protein. This stabilization, as Chamberlin et al. have shown, is not specific; bovine serum albumin, for example, does the job perfectly well, and Summers and Siegel's experiments prove that $E$. coli RNA polymerase is also effective. When they found that the product of gene 1 of $\mathrm{T} 7$ transcribes 17 DNA in vitro in the presence but not in the absence of $E$. coli RNA polymerase, they were detecting this stabilization phenomenon and not, as they suggested, the association of a T7 sigma factor with the core enzyme of $E$. coli.

If the transcription of the genomes of $\mathrm{T} 7$ and $\mathrm{T} 4$ is 80 different, speculations about the role of sigma factors in eukaryotic cells will have to be taken with packets, not pinches, of salt. 